OPEN ACCESS

Edited by:

Peng $\mathrm{He}$,

Guizhou University, China

Reviewed by:

Kai Lu,

Fujian Agriculture and Forestry

University, China

Guan-Heng Zhu,

University of Kentucky, United States

${ }^{*}$ Correspondence:

Peiying Hao

haopeiy@163.com

Xiaoping Yu

yuxiaoping19630306@163.com

Specialty section:

This article was submitted to Invertebrate Physiology, a section of the journal

Frontiers in Physiology

Received: 29 September 2019

Accepted: 24 December 2019

Published: 31 January 2020

Citation:

Yu F, Hao P, Ye C, Feng Y, Pang K and YuX (2020) NIATG1 Gene Participates in Regulating Autophagy and Fission of Mitochondria

in the Brown Planthopper, Nilaparvata lugens. Front. Physiol. 10:1622. doi: 10.3389/fphys.2019.01622

\section{NIATG1 Gene Participates in Regulating Autophagy and Fission of Mitochondria in the Brown Planthopper, Nilaparvata lugens}

\author{
Feifei Yu, Peiying Hao*, Chenglong Ye, Yalin Feng, Kun Pang and Xiaoping Yu* \\ Zhejiang Provincial Key Laboratory of Biometrology and Inspection \& Quarantine, College of Life Sciences, China Jiliang \\ University, Hangzhou, China
}

Autophagy plays multiple roles in regulating various physiological processes in cells. However, we currently lack a systematic analysis of autophagy and the autophagyrelated gene 1 ATG1 in the brown planthopper (BPH, Nilaparvata lugens), one of the most destructive of the insect pests of rice. In this study, the full-length cDNA of an autophagy-related gene, NIATG1, was cloned from BPH. Real-time qPCR (RT-qPCR) revealed that this NIATG1 gene was expressed differently across developmental stages, at higher levels in nymphs but lower levels in adults. RNA interference with dsNIATG1 significantly decreased the mRNA level of the target gene to $14.6 \%$ at day 4 compared with that of the dsGFP control group. The survival of the dsNIATG1-treated group decreased significantly from day 4 onward, dropping to $48.3 \%$ on day 8 . Examination using transmission electron microscopy (TEM) showed that epithelial cells of the BPH's midgut in the dsNIATG1-treated group had less autophagic vacuoles than did the dsGFP control, and knockdown of NIATG1 clearly inhibited the starvation-induced autophagy response in this insect. RNA interference of NIATG1 upregulated the NIFis1 gene involved in mitochondrial fission, leading to reductions in mitochondrial width and area. Furthermore, knockdown of NIATG1 also decreased the ATP content and accumulation of glycogen. Together, these results demonstrate that the NIATG1 gene participates in regulating autophagy and fission of mitochondria in the brown planthopper, making it a potentially promising target for pest control given its key role in autophagy, including maintaining the normal structure and function of mitochondria.

Keywords: autophagy, autophagy-related gene, mitochondrion, mitophagy, Nilaparvata lugens, pest control, RNA interference, survival

\section{INTRODUCTION}

Autophagy is a process of bulk or selective degradation that depends on lysosomes in cells (Van der Vaart et al., 2008). Autophagy plays a pivotal role in maintaining physiological homeostasis and the survival of cells by removing soluble macromolecules from the cytoplasm, organelles, and microorganisms in the cell (Van der Vaart et al., 2008; Jing and Lim, 2012; Zhan et al., 2018). Autophagy may be induced through chemical pesticides, nutrient deficiencies (starvation), hormone stimulation, and ionizing radiation, among other factors (Mcphee and Baehrecke, 2009; 
Wong et al., 2013). Furthermore, both selective and non-selective autophagy exist. The selective pathway targets organelles that are surplus or damaged, such as the mitochondria, endoplasmic reticulum, and ribosome. The non-selective pathway is always induced by starvation, ensuring the normal operation of cells (Papinski and Kraft, 2016). Autophagy-related genes (ATGs) and proteins are key executive factors in both types of autophagy, having been first found in Saccharomyces cerevisiae (Liu et al., 2005). Since then, at least 30 ATGs have been identified (Mari and Reggiori, 2016).

In insects, autophagy occurs in many physiological and morphological processes, including the remodeling of organs (Franzetti et al., 2012; Khoa and Takeda, 2012; Liu et al., 2013), molting (Franzetti et al., 2016), responding to starvation (Liu et al., 2007; Wu et al., 2011), and inhibiting the infection of pathogens (Cherry, 2009; Yue et al., 2015; Wang et al., 2016; Santana et al., 2019). ATG1 is one of the known ATGs, being essential for the initiation of autophagy, by forming an important complex with other proteins. This complex promotes the initiation of autophagy, integrating and transmitting signals from upstream to downstream of the autophagy pathway (Löffler et al., 2011; Shang and Wang, 2011; Wang and Kundu, 2017; Chun and Kim, 2018).

In Bombyx mori, Drosophila melanogaster, and Spodoptera litura, ATG1 interacts with other autophagy-related proteins and enhances autophagy degradation, and RNA interference of the ATG1 gene leads to a decrease in autophagy levels (Scott et al., 2007; Hou et al., 2008; Casati et al., 2012; Zhang et al., 2016). Yet, according to some studies, mechanisms inducing autophagy could operate differently in different species or under different situations. For example, ATG1 was identified as an essential gene for the onset of autophagy in both yeast and Drosophila (Chew et al., 2015; Ma et al., 2018), whereas it was also found that, in mice, autophagy could be stimulated via a process that does not require the involvement of ATG1/ULK1 (Wong et al., 2013). Similarly, although the importance of ATG1 and its complex has been confirmed in Arabidopsis during this plant's autophagy (Suttangkakul et al., 2011; Li et al., 2014), recently, they were found not to be necessary for activating autophagy under prolonged fixed-carbon stress (Huang et al., 2019). By analyzing the genomes of 40 non-unikonts, Földvári-Nagy et al. (2014) found that some of these genomes do not encode typical ATG1 proteins and that the members of the complex are highly variable, thus indicating that some species possess different autophagy-inducing mechanisms. In sum, the regulation of autophagy is a complex phenomenon, and the role of ATG1 in the mechanism of one species cannot be easily generalized to others. Among the insects, autophagy, including the ATG1 gene, has been well studied, albeit that the focus has been mainly on model systems, such as Drosophila and silkworm. Both of these are completely metamorphosed insects, whose organs, such as mouthparts and midgut, undergo remolding during phases of metamorphosis. Since no external nutrition is relied upon during the pupal stage, autophagy is thought to be necessary to remobilize nutrients stored in the pupa. By contrast, the brown planthopper (BPH, Nilaparvata lugens) is an insect that is incompletely metamorphosed and that does not pass through a pupal stage in its life cycle, so its piercing-sucking mouthparts and its mode of feeding are unchanged for its entire lifetime. Further, the BPH only feeds on rice plants, mainly sucking their phloem sap with its piercing-sucking mouthparts; hence, food ingested by the $\mathrm{BPH}$ differs considerably from that obtained by insects with chewing or scratching mouthparts. Therefore, in terms of modes of feeding behavior and metamorphosis, the BPH clearly differs from the well-studied Drosophila and silkworm. Additionally, $\mathrm{BPH}$ is one of the most destructive insect pests of rice crops (Park et al., 2007; Wang et al., 2008; Liu et al., 2015; Xue et al., 2018), but the functioning of autophagy and its related genes in this insect remain, surprisingly, unknown.

In this study, we cloned the full-length cDNA of the autophagy-related gene NlATG1 and analyzed its expression pattern using real-time qPCR (RT- qPCR). We also explored the function of NIATG1 by knocking down its expression via the RNA interference (RNAi) technique. Our results showed that applying RNAi to NlATG1 inhibited autophagy and glycogen metabolism and decreased the ATP content and survival of BPH. Therefore, the NlATG1 gene is required for autophagy and is a promising target for developing a novel BPH control strategy.

\section{MATERIALS AND METHODS}

\section{Insect Organisms}

The BPH insects used in this study were maintained for approximately 100 generations on the susceptible rice variety TN1. All these $\mathrm{BPH}$ insects were reared under the same conditions, at $26 \pm 2{ }^{\circ} \mathrm{C}$, with humidity of $80 \pm 5 \%$ and under a 12-h/12-h light/dark photoperiod.

\section{RNA Extraction and First-Strand cDNA Synthesis}

Nymphs (1st to 5th instar) and adults (1-9 days after eclosion) were collected and immediately frozen in liquid nitrogen, using 10 adults or 30-50 nymphs per replicate. Total RNA was extracted using the MiniBEST Universal RNA Extraction Kit (TAKARA, Tokyo, Japan), according to the manufacturer's instructions. The RNA was then quantified with a Nanodrop 2000 system (Thermo, Wilmington, DE, United States) and its integrity and purity determined by electrophoresis in a $1 \%$ agarose gel. The first strand of cDNA was reverse transcribed, using $1 \mu \mathrm{g}$ of total RNA as the template, with the PrimeScript RT reagent Kit with gDNA Eraser (TAKARA, Tokyo, Japan), which removes genomic DNA in $2 \mathrm{~min}$ at $42^{\circ} \mathrm{C}$ before cDNA synthesis.

\section{Cloning of the Full-Length cDNA of the ATG1 Gene}

According to the transcriptome sequence we obtained, part of the ATG1 gene's core sequence in BPH was selected and identified on the NCBI website via sequence alignment. The PCR primers NlATG1-F and NlATG1-R (Supplementary Table S1) were designed using Primer Premier 5.0 software, and the synthesized first-strand cDNA served as the template for PCR amplifications. This amplification reaction system consisted of 
$50 \mu \mathrm{L}(25 \mu \mathrm{L}$ of PCR Mix, $2 \mu \mathrm{L}$ each of $10 \mu \mathrm{mol} / \mathrm{L}$ positive and negative primers, $2 \mu \mathrm{L}$ of cDNA template, and $19 \mu \mathrm{L}$ of $\mathrm{ddH}_{2} \mathrm{O}$ ). The $\mathrm{PCR}$ reaction procedure had the following conditions: $94^{\circ} \mathrm{C}$ for $4 \mathrm{~min}, 30$ amplification cycles $\left(94^{\circ} \mathrm{C}\right.$ for $30 \mathrm{~s}, 55^{\circ} \mathrm{C}$ for $30 \mathrm{~s}$, and $72^{\circ} \mathrm{C}$ for $3 \mathrm{~min}$ ), and $72^{\circ} \mathrm{C}$ for $10 \mathrm{~min}$. Each PCR amplification product was separated by $1 \%$ agarose gel electrophoresis, and the DNA fragment of interest was recovered with the MiniBEST Agarose Gel DNA Extraction Kit (TAKARA, Tokyo, Japan), cloned into the pMD18-T vector, and then transformed into competent cells of Escherichia coli JM109. The positive clones were selected and sent to the Sunny Biotechnology Company (Shanghai, China) for sequencing. Sequencing results were verified by running alignments with the original sequences, performed in DNAMAN software.

To clone the full-length cDNA of the ATG1 gene, $5^{\prime}$ RACE (rapid amplification cDNA ends) and 3'-RACE was carried out using the BD SMART ${ }^{\mathrm{TM}}$ RACE cDNA Amplification Kit (Clontech, Palo Alto, CA, United States). The outer primers (i.e., NlATG1-5'RACE outer/NlATG1-3'RACE outer) and the internal primers (i.e., NlATG1-5'RACE inner/NlATG13'RACE inner) were designed separately (Supplementary Table S1). According to the RACE kit instructions, the $3^{\prime}$ and $5^{\prime}$ ends of the target gene were amplified via nested PCR, and the ensuing product was separated by agarose gel electrophoresis. The DNA fragment of interest was recovered, cloned into the pMD18-T vector, and transformed into competent cells of E. coli JM109. The positive clones were selected and sent to Sunny Company for sequencing. These sequencing results were then spliced using DNAMAN software to obtain the full-length cDNA sequence of ATG1 (NlATG1). The full-length cDNA of NlATG1 was verified by PCR, with the primers NlATG1-FL-F and NlATG1-FL-R (Supplementary Table S1) designed for the two ends of the spliced sequence, respectively.

\section{Sequence Analysis of NIATG1}

After the full-length cDNA of NlATG1 was cloned, its open reading frame (ORF) was found using the ORF finder tool ${ }^{1}$. Next, an NCBI BLASTX search was performed for the amino acid sequence homology alignment. The molecular weight and theoretical isoelectric point of a protein were analyzed by ExPASy ${ }^{2}$, and its signal peptide was predicted using the online SignalP 4.1 server $^{3}$. All protein domains were predicted in the PROSITE database ${ }^{4}$. A phylogenic tree of ATG1 was constructed based on the amino acid sequences from the $\mathrm{BPH}$ and other species by using the neighboring-joint (NJ) method in MEGA 5.0. Testing of this phylogeny was done by bootstrapping $(n=1000$ replications), for which percentage values larger than 50 were shown on each node of the tree. Multiple sequence alignments were performed in DNAMAN software.

\footnotetext{
${ }^{1}$ https://www.ncbi.nlm.nih.gov/orffinder/

${ }^{2}$ http://web.expasy.org/protparam/

${ }^{3}$ http://www.cbs.dtu.dk/services/SignalP/

${ }^{4}$ http://prosite.expasy.org/
}

\section{Real-Time-qPCR Analysis of Gene Expression}

To analyze NlATG1 gene expression in BPH during its developmental stages, nymphs (1st, 2nd, 3rd, 4th, and 5th instar) and adults (females and males at 1, 3, 5, 7, and 9 days post-eclosion) were sampled separately, with 10 adults or 30-50 nymphs used per replicate. To analyze NlATG1 gene expression in different parts or tissues, 30 individuals of 5 th instar nymphs were sampled and dissected (head, thorax, midgut, ovary, and fat body).

RNA extraction and reverse transcription of the first strand of cDNA was carried out as described in subsection 2.3 above. The genes' specific primers for the RT-qPCR can be found in Supplementary Table S1, and the RPS11 gene of BPH served as an internal reference to detect the relative expression level of the target genes in each sample (Yuan et al., 2014). Three independent biological replicates and three technical replicates were set up in the experiment. The relative expression level of a given gene was calculated using the $2^{-\Delta} \Delta \mathrm{Ct}$ method (Livak and Schmittgen, 2001).

\section{Double-Stranded RNA Synthesis}

Primers of dsNlATG1-F and dsNlATG1-R (Supplementary Table S1) for the synthesis of double-stranded RNA interference fragments were designed based on the full-length cDNA sequence (i.e., from 272 to 993, $722 \mathrm{bp}$ ). A protective base (GGATCC) and a T7 promoter (TAATACGACTCACTATA) were added to the $5^{\prime}$ end of the specific primer and cloned into pMD18-T vector, which was then transferred into JM109 for sequencing. In order not to affect the transcription level after RNAi, the interference fragment did not include the fragment for RT-qPCR. The dsRNA was synthesized by following the instructions of the MEGAscript ${ }^{\circledR}$ T7 High Yield Transcription Kit (Ambion, Austin, TX, United States). To purify the dsRNA, $30 \mu \mathrm{L}$ of $\mathrm{ddH}_{2} \mathrm{O}$ and $30 \mu \mathrm{L}$ of the $\mathrm{LiCl}$ Precipitation Solution were added to the dsRNA reaction system. This was allowed to sit at $-20^{\circ} \mathrm{C}$ for $1 \mathrm{~h}$, after which it was centrifuged at $4^{\circ} \mathrm{C}$, $11,000 \mathrm{r} / \mathrm{min}$ for $15 \mathrm{~min}$, and the supernatant removed; $1 \mathrm{~mL}$ of $70 \%$ ethanol (prepared with DEPC water) was added to the solution, centrifuged again, and the ethanol removed. The dsRNA products were then resuspended in $20 \mu \mathrm{L}$ of $\mathrm{dd}_{2} \mathrm{O}$, verified, and kept at $-80^{\circ} \mathrm{C}$ for the following RNA interference experiments. Using the same method, dsGFP was prepared with the primers dsGFP-F and dsGFP-R (Supplementary Table S1), according to the sequence (from 1155 to 1811, $657 \mathrm{bp}$ ) of a green fluorescent protein GFP (GenBank accession number: MF169984.1).

\section{dsRNA Microinjection}

Nymphs (2nd instar) were microinjected with dsNlATG1 as the treatment, for which dsGFP served as the control; each group consisted of $60 \mathrm{BPH}$ individuals. The final concentration of purified dsRNA was $5 \mu \mathrm{g} / \mu \mathrm{L}$. The microinjection site was located at the coxal cavity of the mesofoot (Liu et al., 2010). Any dead individuals were removed, and the survival was recorded daily. After microinjecting them, the nymphs were 
placed onto the susceptible rice variety $\mathrm{TN} 1$ and reared under the same conditions $\left(26 \pm 2{ }^{\circ} \mathrm{C}\right.$, humidity of $80 \pm 5 \%, 12-\mathrm{h} / 12-\mathrm{h}$ light/dark photoperiod).

In addition, a parallel RNA interference treatment was set up to analyze the expression level of the NlATG1 gene and mitochondrial fission gene NlFis1 and fusion gene NlMarf, with three replicates for each. Each group was sampled every 2 days, and six individuals were randomly selected and frozen in liquid nitrogen and kept at $-80^{\circ} \mathrm{C}$ for subsequent RNA extractions. The RT-qPCR methods, primers, and reaction conditions were the same as those described in subsection 2.5.

\section{Sample Processing Procedures of TEM}

The BPH individuals were dissected under a microscope and first fixed overnight with $2.5 \%$ glutaraldehyde, then fixed again with $1 \% \mathrm{OsO}_{4}$ for $1 \mathrm{~h}$ after washing with PBS. Samples were dehydrated by a graded series of ethanol $(30,50,70$, 80, 90, 95, and 100\%) and sectioned using a Leica EM UC7 ultratome after being embedded. Sections were stained by uranyl acetate and alkaline lead citrate for 5-10 min and observed using transmission electron microscopy (TEM) under a Hitachi Model H-7650.

\section{ATP Content Measurement}

Approximately $20 \mathrm{mg}$ of a BPH sample treated with dsNlATG1 or dsGFP for 4 days was homogenized with a $150-\mu \mathrm{L}$ lysis buffer following the instructions of the ATP Assay Kit (Beyotime, Shanghai, China) and centrifuged at $12000 \times g$ and $4^{\circ} \mathrm{C}$ for $5 \mathrm{~min}$. The supernatant $(20 \mu \mathrm{L})$ was removed for the detection of ATP in a black 96-well plate. The protein concentration was measured simultaneously using the BCA Protein Assay Kit (Beyotime, Shanghai, China) to enable the final calculation of ATP content. Three independent biological replicates and three technical replicates were used to measure ATP in this way.

\section{Statistical Analysis}

The software programs SPSS 20.0 and GraphPad Prism 6.0 were used to analyze the data and draw the figures. ImageJ was used to measure the width and length of mitochondria. All values were presented as the mean $\pm \mathrm{SD}$ of at least three independent biological replications. Student's $t$-tests were used to compare means of two groups of samples; one-way ANOVA followed by Tukey's post hoc test was used to compare three or more group means of samples.

\section{RESULTS}

\section{Full-Length cDNA Cloning and Sequence Characterization of NIATG1}

The RACE results showed that the NIATG1 gene had a full-length cDNA of 2502 bp. It was predicted that NlATG1 contains a 2040bp ORF (GenBank accession number: MF062504) and encodes a hypothetical protein of 679 amino acids. The NlATG1 protein was estimated to have a molecular weight of $74.3 \mathrm{kDa}$ with an isoelectric point of 8.31. No signal peptide was found for it.
Phylogenetic analysis revealed that ATG1 had homology with different species. The ATG1 protein of BPH was quite similar to Cimex lectularius (Hemiptera), Halyomorpha halys (Hemiptera), and Recilia dorsalis (Homoptera); among them, the most closely related amino acid sequence to NlATG1 is from $R$. dorsalis, with 95\% shared identity (Figure 1A). Multiple amino acid sequence alignment from those four insects showed that all sequences harbor a protein kinase domain, albeit with some differences among them (Figure 1B). The protein kinase domain (PS50011) of NlATG1 contains a protein kinase ATPbinding region (at positions 15 through 39), a serine/threonine protein kinases activity site (at positions 130 through 142), and a proton acceptor (at position 134) (Figure 1C). Based on analysis of its structure, the physiological function of ATG1 protein is predicted to be similar to its homologous counterparts in other insect species.

\section{Expression Pattern of NIATG1 in BPH}

Using RT-qPCR, the expression pattern of NlATG1 was investigated in $\mathrm{BPHs}$ raised on susceptible $\mathrm{TN1}$ rice. These results demonstrated that NIATG1 was expressed in different developmental stages, but at higher levels in nymphs and lower levels in both male and female adults (Figure 2A). Generally, the expression level in adults began to decrease after eclosion, reaching its lowest level at day 5, after which it increased slightly over the following days. Additionally, the transcription level of NlATG1 was not correlated with either macropterous or brachypterous wing forms, also showing no correlation with females or males in the adult stage (Supplementary Figure S1). Concerning the 5th instar nymphs, NlATG1 had different expression levels in them in different tissues, being higher in the head, midgut, ovary, and fat body but lowest in the thorax (Figure 2B). The TEM revealed that autophagy did occur in certain tissues, such as the midgut and fat body, with some mitochondria found in autophagosomes undergoing mitophagy (Figures 2C,D).

\section{RNAi of NIATG1 Reduced the Survival of BPH Juveniles and Adults}

The qPCR results showed that the expression level of NlATG1 was significantly knocked down after RNA interference, decreasing to $40.5,14.6,26.4$, and 41.2 of the dsGFP control group at days $2,4,6$, and 8 , respectively (Figure 3A). Hence, the efficacy of RNA interference peaked at day 4 but then diminished over time. The expression level of NlATG1 in different tissues was also significantly knocked down at day 4, decreasing to 31.1, 32.2, 14.3, and $20.3 \%$ of the control group in the head, thorax, midgut, and fat body, respectively (Figure 3B).

The injection of dsNlATG1 caused a significant decrease in $\mathrm{BPH}$ survival from day 4. At day 8 , the survival of the treated group had decreased to $48.3 \%$, whereas the control group maintained a higher survival of $78.3 \%$ (Figure 3C). It is worth noting that RNAi of dsNlATG1 considerably lowered the survival of female adults, such that all dsNlATG1-treated females had died within 8 days after 


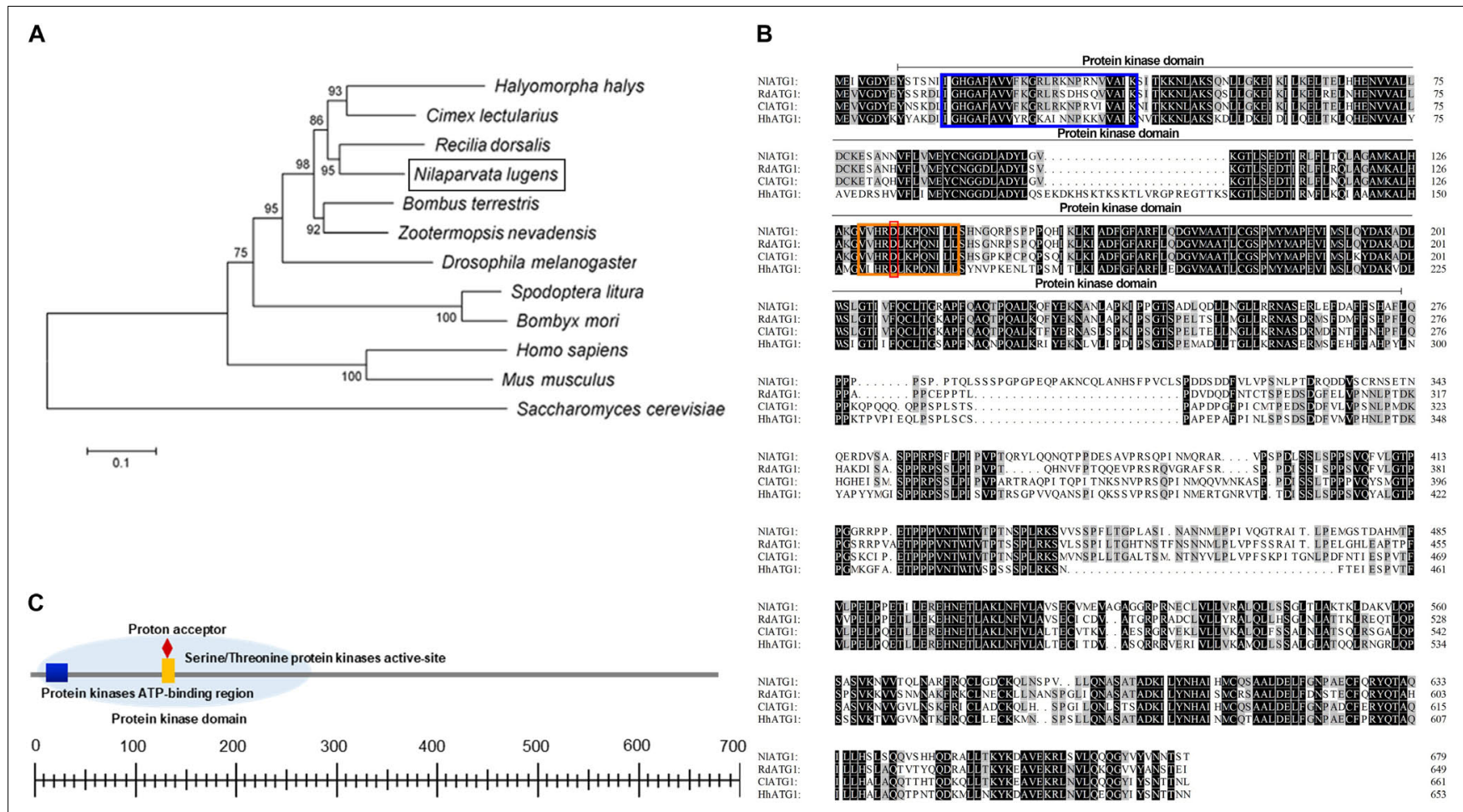

FIGURE 1 | Analysis of the ATG1 protein sequence. (A) Phylogenic tree of ATG1 from the brown planthopper (BPH, Nilaparvata lugens) and other insect species based on amino acid sequences and using the neighboring-joint method. The tree was constructed in MEGA 5.0, and the phylogeny was tested with the bootstrap method (1000 replications); percentage values $>50$ are shown on each node. NIATG1 is shown in a black box. Accession numbers for the sequences of the other species are as follows: Halyomorpha halys, XP_014270311.1; Cimex lectularius, XP_014254421.1; Recilia dorsalis, ATV91623.1; Bombus terrestris, XP_003394615.1; Zootermopsis nevadensis, XP_021941781.1; Drosophila melanogaster, NP_001163433.1; Spodoptera litura, AJE75846.1; Bombyx mori, NP_001296475.1; Homo sapiens, NP_003556.1; Mus musculus, NP_038909.3; Saccharomyces cerevisiae, KZV11049.1. (B) Comparison of four amino acid sequences from N. lugens, H. halys, C. lectularius, and R. dorsalis. Amino acids identical in all four proteins are marked in black, with three identical amino acids marked in gray. Blue box, protein kinase ATP-binding region; orange box, serine/threonine protein kinases activity site; red box, proton acceptor. (C) Structural analysis of the protein domain from NIATG1. The scaled line below indicates the length of the amino acid.

eclosion, while the control group's survival was higher at $66.7 \%$ (Figure 3D). In addition, some BPHs died of molting failure (Figures 3E,F).

\section{RNAi of NIATG1 Inhibited Autophagy in Epithelial Cells of the Midgut in BPH}

The TEM results showed that in normally feeding BPHs, the epithelial cells of their midgut underwent basic autophagy with several autophagic vacuoles or autophagic compartments, including mitochondrial autophagy (mitophagy) (Figures 4AC). In stark contrast, almost no vacuoles were observed in those cells interfered with by dsNlATG1 (Figures 4D-F). Further, RNA interference with dsNlATG1 also affected the metabolism of glycogen, such that glycogen accumulated in the midgut cells (Figure 4F).

To further validate the effect of RNA interference of NlATG1 upon autophagy, the BPH was first injected with dsRNA and then left to starve. TEM examinations showed that autophagy was evidently induced in the starving midgut cells of dsGFP-treated BPH individuals (Figures 5C,D), with similar autophagy vacuoles as in starving non-treated individuals (Figures 5A,B). By contrast, autophagy was clearly inhibited in the dsNlATG1-treated group, with almost no vacuoles visibly formed in the midgut cells even under starvation conditions (Figures 5E,F).

\section{Effects of NIATG1 RNAi on Mitochondrial Morphosis}

TEM graphs showed that the mitochondria in cells treated with dsGFP were generally long or short and rod-like, sectioned into different shapes (e.g., spherical or oval and tubular; Figure 4C). Compared with the dsGFP group, the shape of mitochondria treated with dsNlATG1 did not change much, but they did become narrower (Figure 4F). The width (diameter) of mitochondria in the dsNlATG1treated groups $(0.14 \mu \mathrm{m})$ was approximately $41 \%$ that of the dsGFP control group $(0.34 \mu \mathrm{m})$ (Figure 6A). Accordingly, the section areas of the mitochondria in the dsNlATG1treated groups were also reduced (Figure 6B). Moreover, mitochondrial width and area also decreased in the $\mathrm{BPH}$ group first treated with dsNlATG1 and then left to starve (Figures 7A,B). Collectively, these results indicated the mitochondria treated with dsNlATG1 became narrower or smaller than the control. 

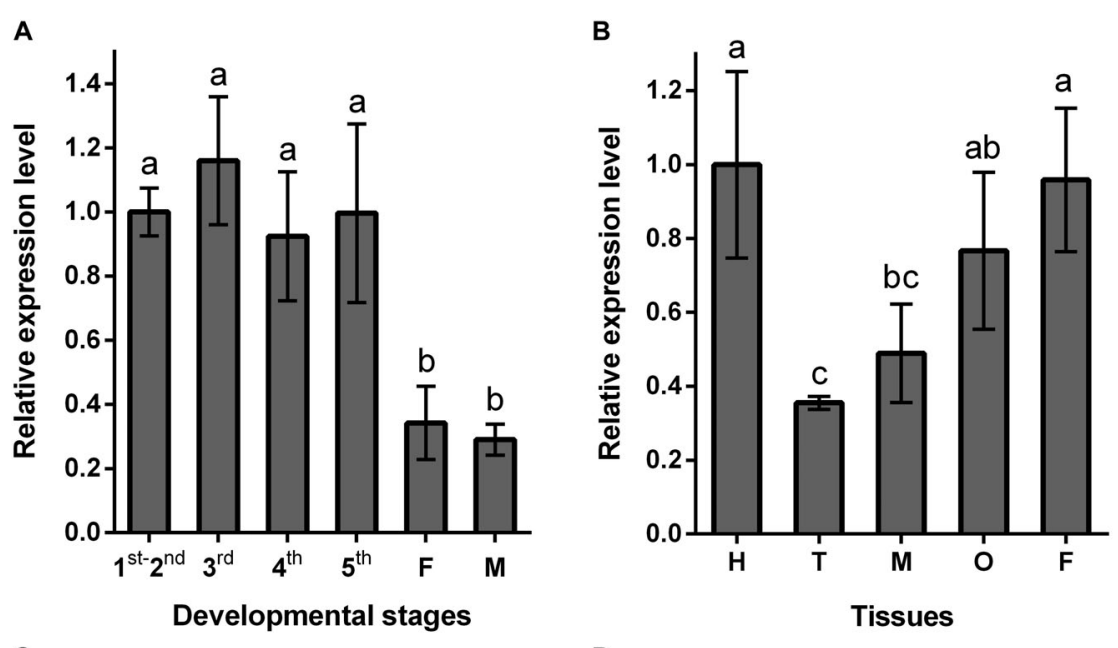

C

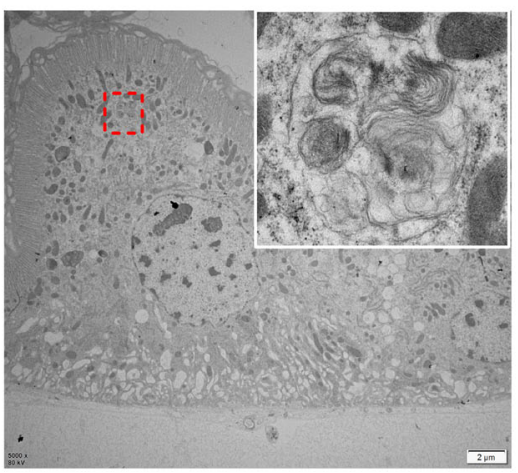

D

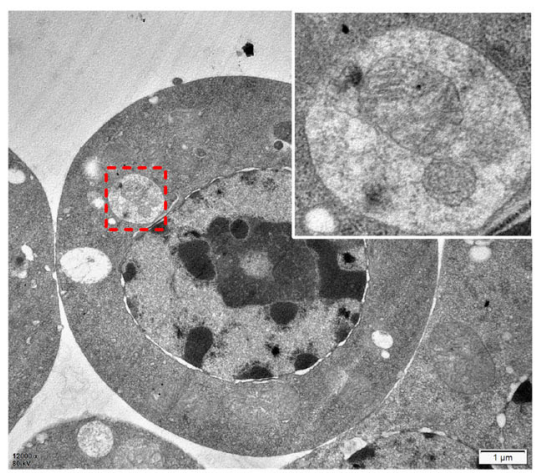

FIGURE 2 | Expression patterns of NIATG1 in BPH. (A) Expression patterns of NIATG1 at different developmental stages. The mRNA level was normalized relative to the RPS11 levels, and the reference was the mRNA level of 1st-2nd instar nymphs. 1st-5th: BPH nymphs from 1st instar to 5th instar, F: female adults, M: male adults. (B) Relative expression of NIATG1 in different insect tissues. H: head, T: thorax, M: midgut, O: ovary, and F: fat body. The reference used was the mRNA level of the head. Relative gene expression was compared by Tukey's test, for which a, b, and c on the bars indicate significant differences among each sample $(p<0.05)$. (C,D) Autophagy occurred in midgut cells and fat body cells examined under a transmission electron microscope. The red dotted box denotes the autophagic vacuoles that are enlarged in the top right-hand corner. Scale bars: $2 \mu \mathrm{m}$ in (C); $1 \mu \mathrm{m}$ in (D).

\section{RNAi of NIATG1 Upregulated the Mitochondrial Fission Related Gene NIFis1}

To better understand how the narrower mitochondria formed, the expression levels of mitochondrial fission gene NlFis1 and fusion gene NlMarf were both analyzed. According to the RT-qPCR, the mRNA level of NlFis1 increased significantly in the dsNlATG1-treated groups, increasing by $117.2 \%$ at day 8 when compared with that of the control (Figure 8A). However, the mRNA transcription level of NlMarf showed no significant change (Figure $\mathbf{8 B}$ ). Therefore, the narrower or smaller mitochondria should have arisen from mitochondrial fission, regulated by the NlFis1 gene.

\section{RNAi of NIATG1 Decreased the ATP Content}

Because ATP is mainly synthesized in the mitochondria, ATP content can reliably reflect the functioning of this organelle. To examine whether the RNAi of NlATG1 affected mitochondrial function in ATP synthesis, the ATP content was determined. This showed that injection of dsNlATG1 significantly decreased the ATP levels, in that the ATP content in the treated groups $\left(0.4 \mathrm{nM} \mu \mathrm{g}^{-1}\right)$ was approximately $36 \%$ that of the dsGFP control group (1.1 nM $\left.\mu \mathrm{g}^{-1}\right)$. Hence, RNAi of NlATG1 altered mitochondrial functioning in the BPH's synthesis of ATP (Figure 8C).

\section{DISCUSSION}

In this study, we identified an autophagy-related gene, NlATG1, and demonstrated that it is essential for autophagy in an insect pest of rice, the brown planthopper $(\mathrm{BPH})$. We found that RNA interference targeting the ATG1 gene significantly inhibited the autophagy and decreased the survival of $\mathrm{BPH}$, especially reducing the survival of its female adults. The number of females is an important factor affecting the insect's population size and growth, so we suggest that it offers a promising approach to controlling BPH by inhibiting ATG1 gene 
A

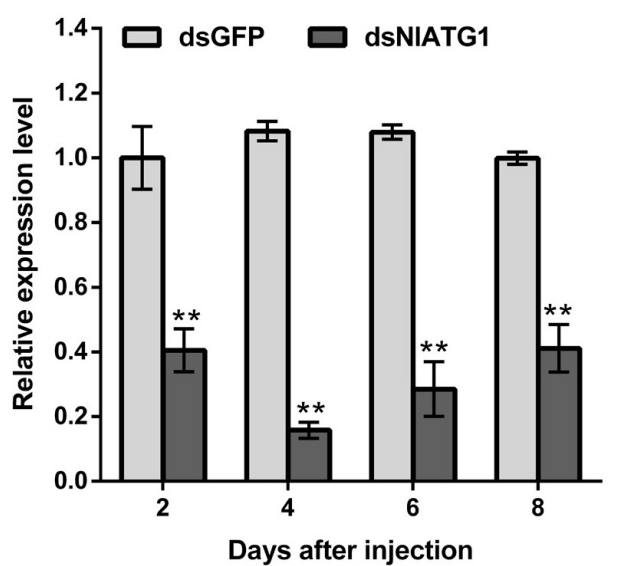

C

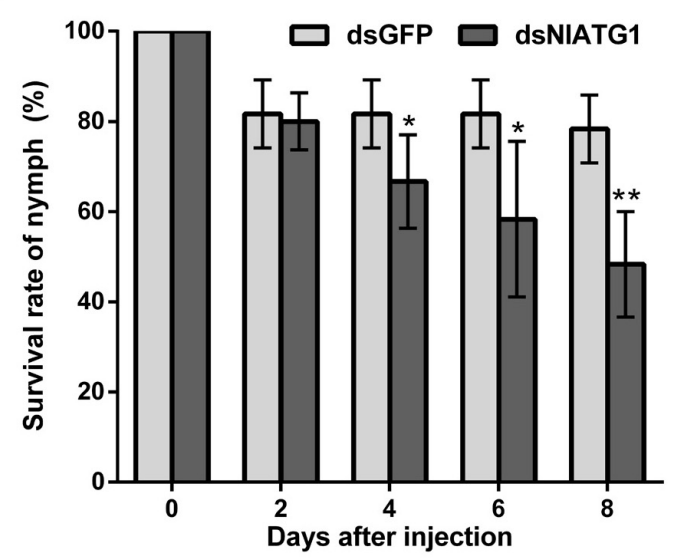

E

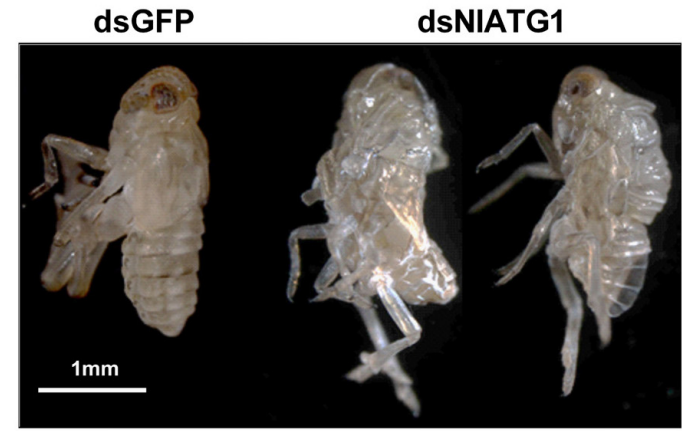

B

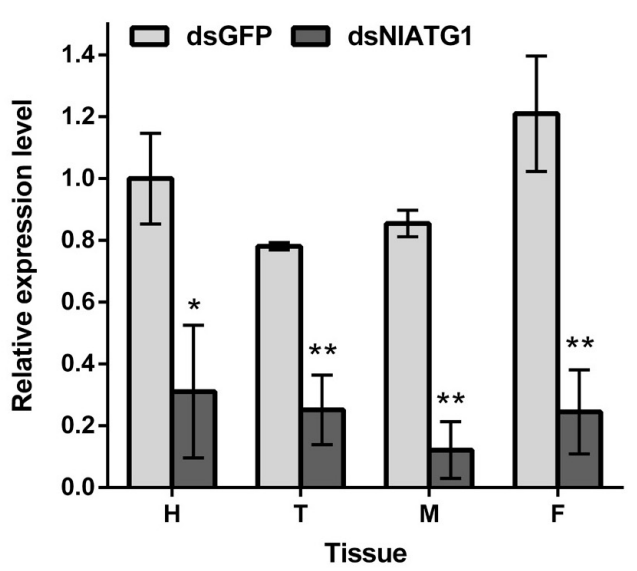

D

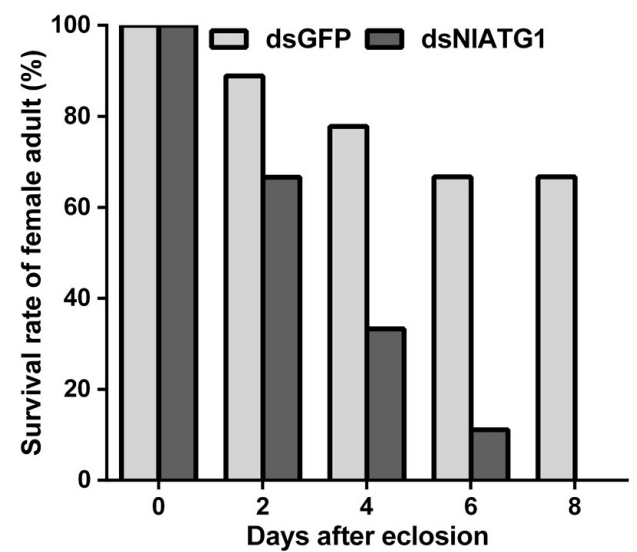

$\mathbf{F}$

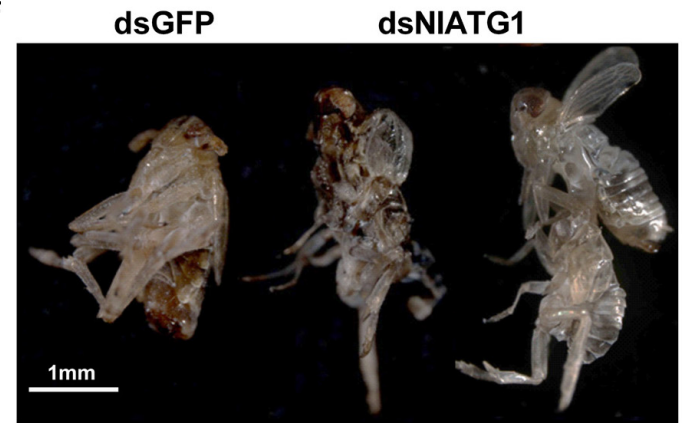

FIGURE 3 | Effects of NIATG1 gene RNA interference on BPH. (A,B) Effect on the mRNA expression level of NIATG1 microinjected with dsRNA at different treatment times (A) and in different tissues at day 4 (B). The reference was the mRNA level of BPH treated with dsGFP at day 2 (A) and head (B). (C,D) Effect of NIATG1 RNAi on the survival rate of BPH nymph (C) and female adult (D). All values are presented as the means \pm SD of three independent replicates $\left({ }^{*} p<0.05,{ }^{* *} p<0.01\right)$. (E,F) Some BPH died of failure in molting and eclosion; scale bars: $1 \mathrm{~mm}$.

expression and autophagy. Furthermore, RNA interference of NlATG1 upregulated the NlFis1 gene involved in mitochondrial fission, leading to reductions in mitochondrial width and area. Knockdown of NlATG1 also decreased the ATP content and accumulation of glycogen. As far as we know, this is the first time that the ATG1 gene, as well as autophagy, has been systematically studied in the incompletely metamorphosed insects such as BPH. Previous studies on insect autophagy were mostly carried out at the cellular level of completely metamorphosed insects, focusing mainly on the regulatory mechanism of autophagy, but they rarely explored its effect on the survival of individuals (Lee et al., 2002; Scott et al., 2007; Casati et al., 2012; Liu et al., 2013; Franzetti et al., 2016; Zhan et al., 2018). Therefore, our work here identified a new potential target, NlATG1, for controlling BPH and sheds fresh light on the autophagy in an incompletely metamorphosed insect, which is of great significance for developing novel RNAibased pest control strategies.

Compared with chemical pesticides, employing an RNAibased strategy is an environmentally friendlier way to control insect pests, since it can be designed specifically to control the 


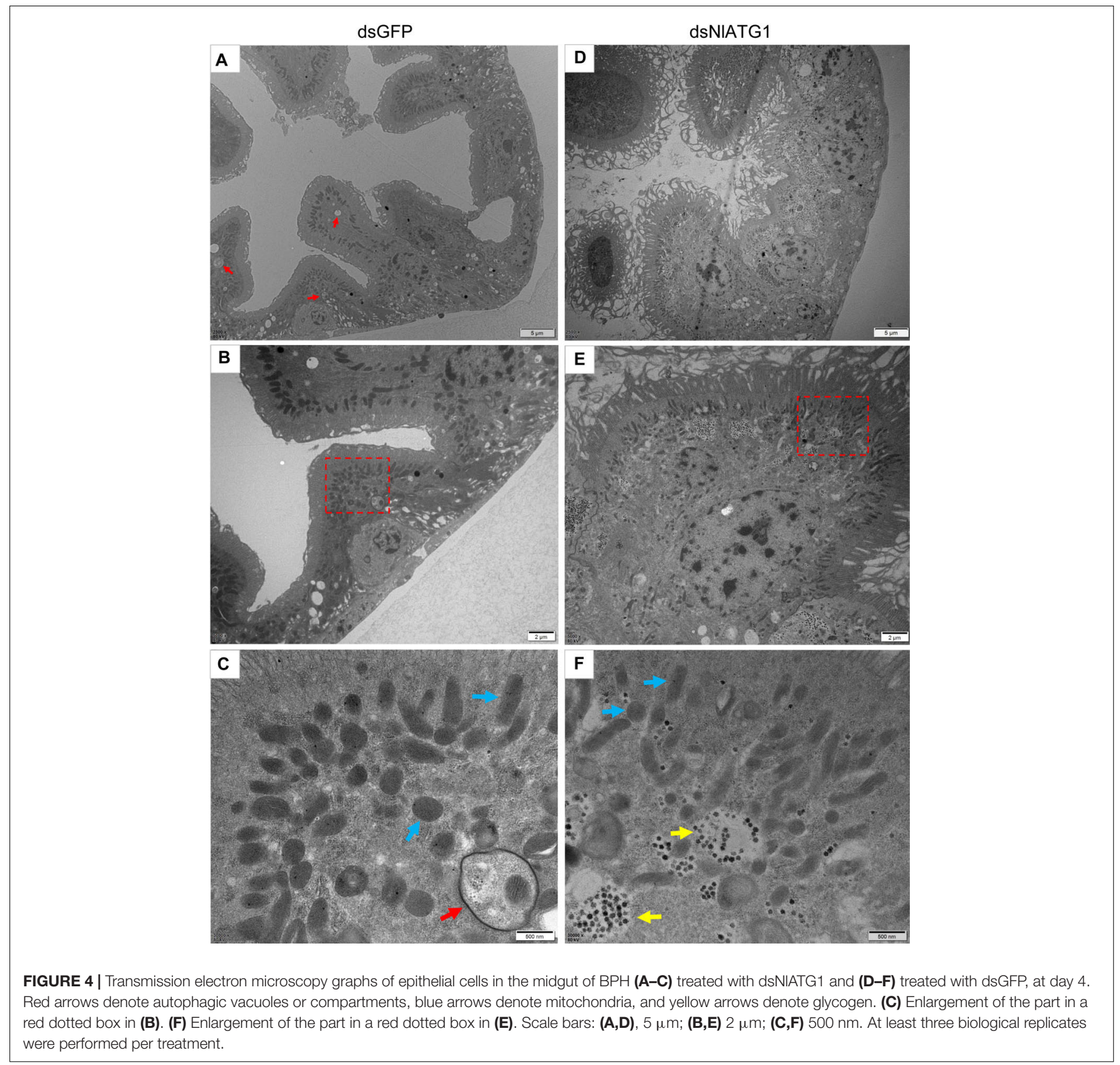

target insect pest without impacting beneficial insects or other animals such as pollinators or pest parasitoids (Scott et al., 2013; Yu et al., 2016; Cooper et al., 2019; Liu et al., 2019). For example, species-specific dsRNAs for Snf7 or V-ATPaseE killed the targeted insects without affecting non-targeted species (Whyard et al., 2009; Bachman et al., 2013; Liu et al., 2019). In our study, phylogenetic analysis showed that the NlATG1 protein of BPH closely resembles the ATG1 protein (ATV91623.1) of R. dorsalis. By aligning the mRNA sequence of NlATG1 (MF062504) from BPH and the mRNA sequence of ATG1/ULK1 (MF038047.1) from $R$. dorsalis, two matches were submitted with respective identities of $74 \%$ (8 gaps) and $68 \%$ (28 gaps). According to Scott et al. (2013), single mismatches between the target gene and dsRNA generally impair the RNAi effect; so we think that the sequencing of more and more insect genomes will greatly enhance the possibility of designing suitable dsRNA specifically targeting the NlATG1 gene of BPH without causing off-target effects in the field. Current dsRNA treatment modes, such as feeding, microinjections, spraying, and GMO-based strategies, are popular for insects (Araujo et al., 2006; Turner et al., 2006; Baum et al., 2007; Mao et al., 2007; Wang et al., 2011). However, it remains a formidable task to deliver dsRNA into a piercing-sucking insect in the agricultural industry, and $\mathrm{BPH}$ is no exception. For example, piercing-sucking insects feed on phloem sap while ingesting very little plant tissue, so foliar application of dsRNA by spraying is not likely to trigger an 

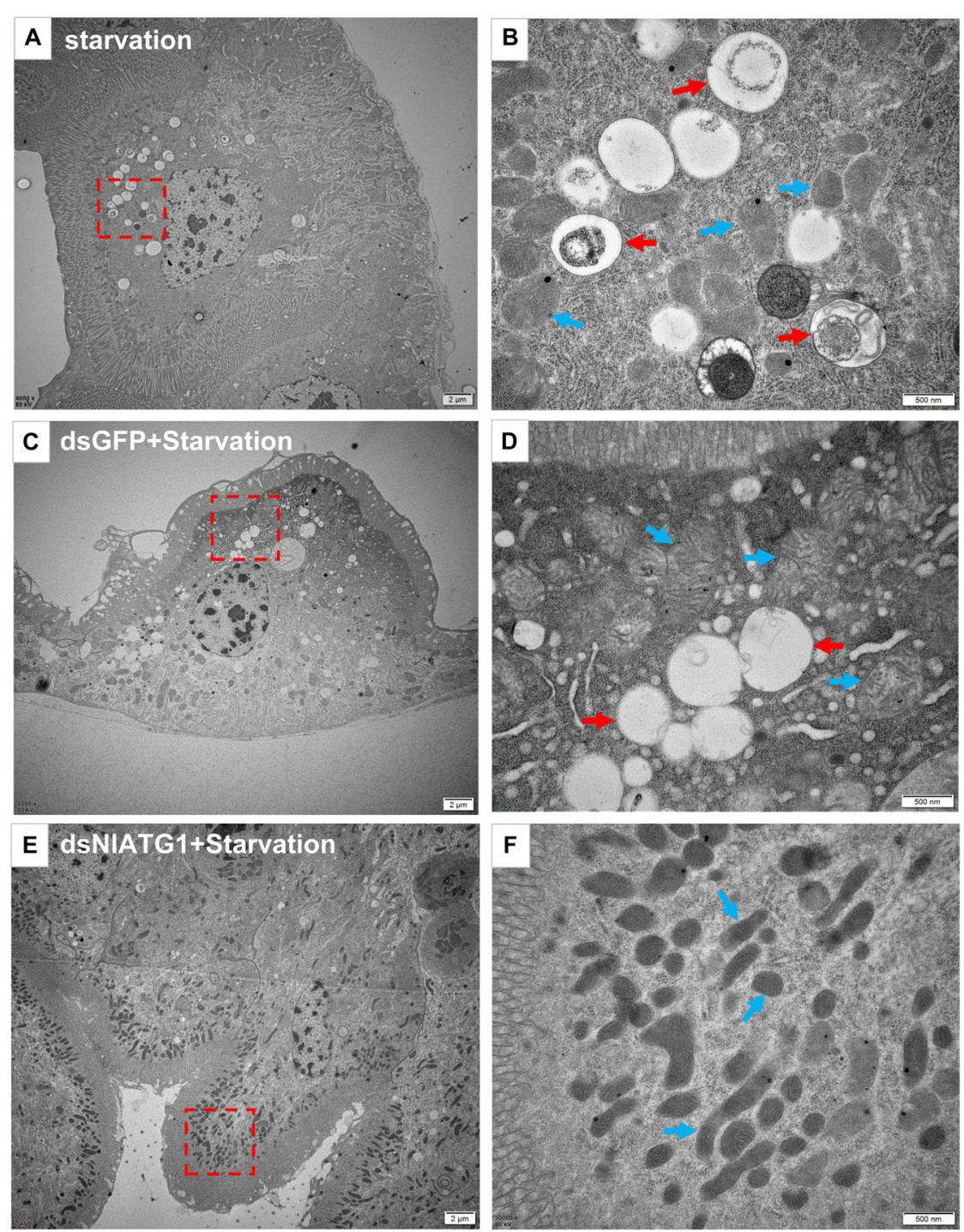

FIGURE 5 | Transmission electron microscopy graphs of midgut cells. (A,B) The control group of the starvation treatment at 48 h; (C,D) starvation treatment at 48 h after injecting dsGFP for 4 days. (E,F) starvation treatment for $48 \mathrm{~h}$ after injecting dsNIATG1 for 4 days. Red arrows denote autophagic vacuoles or compartments, while blue arrows denote the mitochondria. (B,D) and $\mathbf{( F )}$ represent enlargements of the parts indicated by red dotted boxes in $(\mathbf{A}, \mathbf{C})$ and $\mathbf{( E )}$, respectively. Scale bars: (A,C,E) $2 \mu \mathrm{m}$; (B,D,F) $500 \mathrm{~nm}$. At least three biological replicates were used per treatment.

effective RNAi response in them. Microinjection is an effective strategy for delivering dsRNA into many orders of insects, including BPH (Wang et al., 2017; Zhu et al., 2017; Waris et al., 2018; Ge et al., 2019), but this approach is not a feasible strategy for achieving widespread crop protection in the field (Huvenne and Smagghe, 2010; Scott et al., 2013; Liu et al., 2019). Recently, Li et al. (2015) suggested that soaking the root system in dsRNA could be used as a dsRNA-delivery strategy during crop irrigation, which is also suitable for BPH control. Therefore, the next step in our work will focus on how to practically couple the NlATG1 gene with an RNA-based pestcontrol strategy.

We found that autophagy occurred at all developmental stages of BPH, especially during its nymphal stages. RNAi of NlATG1 resulted in mortality of both nymphs and adults but decreased the survival of female adults the most (Figure 3C). It is unclear whether treatment with dsNlATG1 resulted in greater mortality of females than males as nymphs, for it is difficult to distinguish females from males when they are immature. According to our study's RNAi results, autophagy plays multiple roles, not only in molting but also in maintaining the homeostasis of mitochondria and regulating the metabolism of glycogen. Yet it is still uncertain which of these, upon interference, is the main reason for BPH mortality. Therefore, why the RNAi of NlATG1 induced more female adults to die than male counterparts requires further investigation.

The finding in this study that NlATG1 was expressed in different organs/tissues further indicated that this gene should 

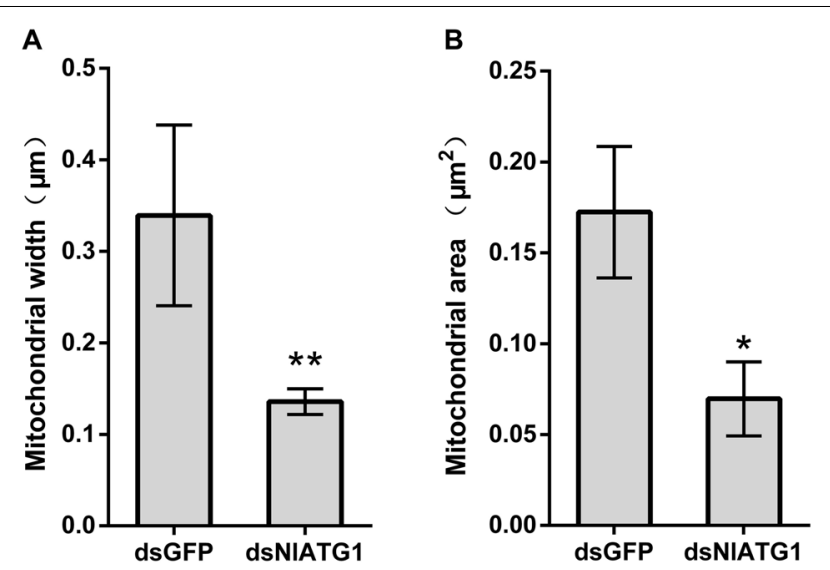

FIGURE 6 | Mitochondrial width and mitochondrial area when treated with dsRNA. (A) mitochondrial width; (B) mitochondrial area. All values are presented as the mean \pm SD of three independent biological replicates $\left({ }^{\star} p<0.05,{ }^{\star \star} p<0.01\right)$. (Approximately 100 mitochondria were measured per replicate).

play multiple roles in BPH. Similarly, in a previous study, BmATG1 was found to be expressed in different tissues of Bombyx mori, especially in its larval midgut during the larval-to-pupal transformation (Casati et al., 2012). Earlier, it was shown that D. melanogaster had great sensitivity to starvation and possible failure in pupation stages under conditions of insufficient DmATG1 (Scott et al., 2007; Hou et al., 2008). However, those studies on the autophagy of complete metamorphosis insects mainly focused on pupa or larval-adult transformation, leaving much information unclear in incomplete metamorphosis insects. Therefore, our findings here for $\mathrm{BPH}$ provided new information on autophagy in the midgut of an incompletely metamorphosing insect. Mitochondria are abundant in the epithelial cells of the midgut, where they supply the large amounts of ATP required for
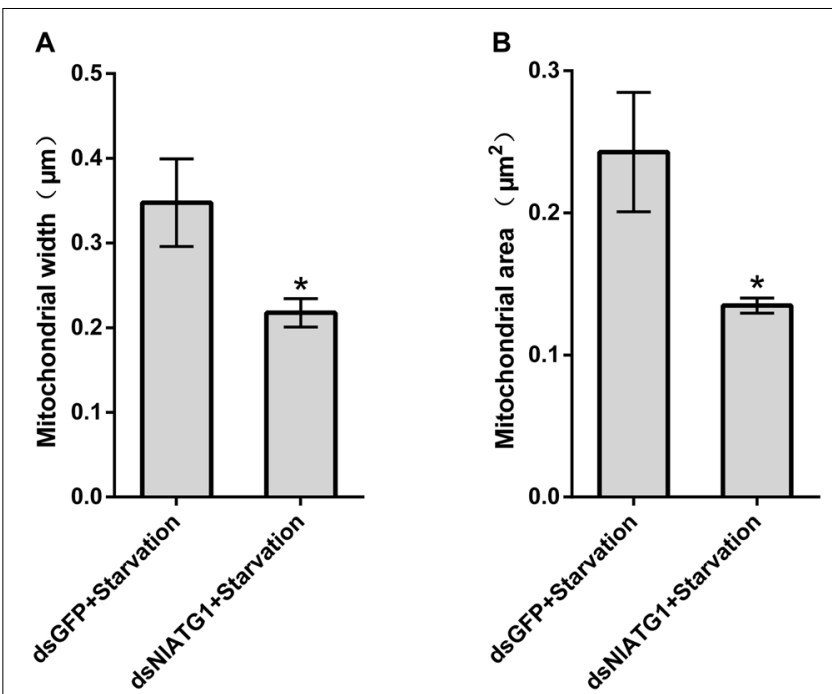

FIGURE 7 | Mitochondrial width and mitochondrial area when treated with dsRNA and starvation. (A) mitochondrial width; (B) mitochondrial area. All values are presented as the means $\pm \mathrm{SD}$ of three independent biological replicates $\left({ }^{\star} p<0.05\right)$. (Approximately 100 mitochondria were measured per replicate).

transporting nutrients from the intestinal lumen to the cytoplasm of the epithelial cell (Manjunatha et al., 2018). From this point of view, the epithelial cell of the midgut offers a good model for analyzing autophagy, and, in particular, mitochondrial autophagy (mitophagy) (Lee et al., 2002; Lőrincz et al., 2017).

Mitochondria maintain their homeostasis through the dynamic equilibrium of fission/fusion (Arun et al., 2016). During this process, the mitochondria periodically exchange proteins, mtDNA, and lipids via rapid and alternating fusion and fission (Twig et al., 2008b). Nevertheless, mitochondrial fission/fusion defects will limit mitochondrial movement, resulting in reduced
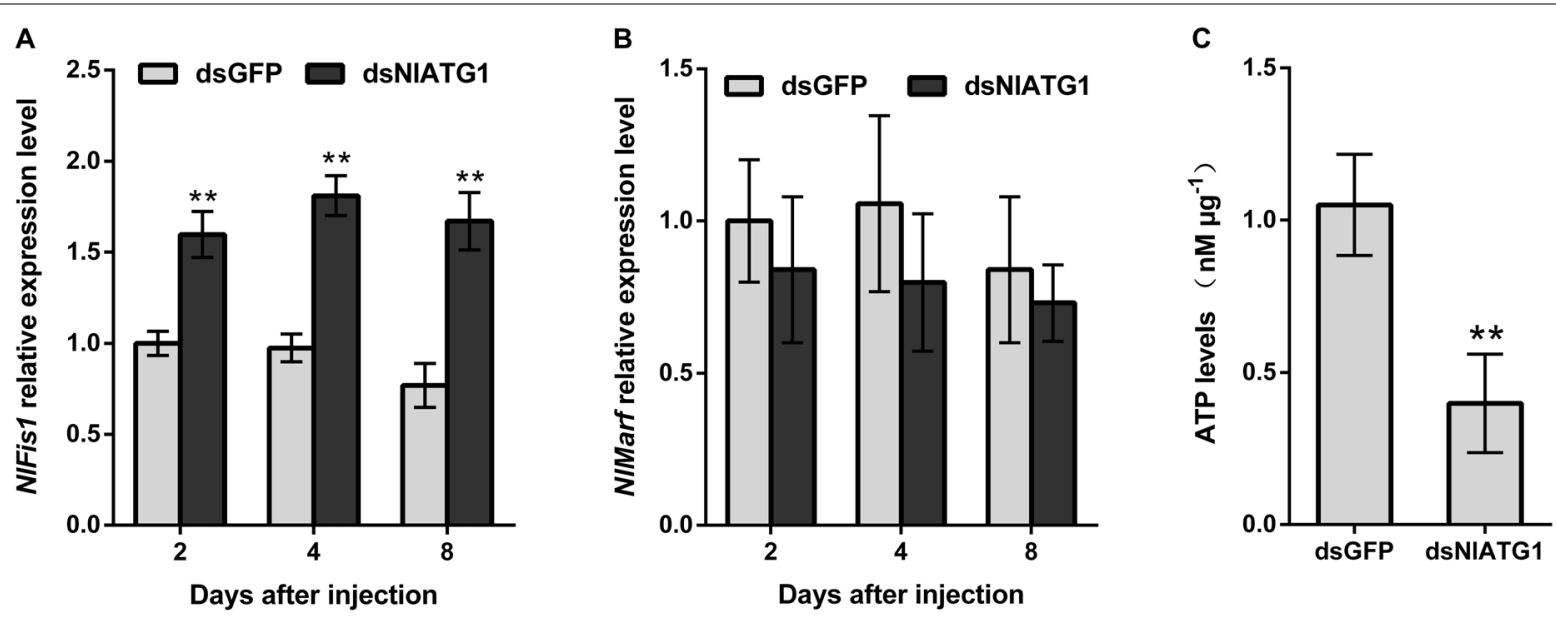

FIGURE 8 | Relative expression level of the mitochondrial fission gene NIFis1 and fusion gene NIMarf, and the ATP content of BPH. (A) Expression level of NIFis 1 gene. (B) Expression level of NIMarf gene. (C) The ATP content. BPH individuals were treated with dsRNA for 4 days. All values are presented as the mean \pm SD of three independent biological replicates $\left({ }^{\star *} p<0.01\right)$. 
energy production and oxidation (Knott and Bossy-Wetzel, 2008). Proteins or mtDNA in mitochondria are easily damaged, so mitochondria must carry out fusion and fission events to facilitate the adequate exchange of vital organelle contents (Twig et al., 2008a). In our study, NlFis1 expression was detected that increased upon NIATG1 interference, but the fusion-related gene NlMarf did not vary significantly, thus indicating that the dynamic balance of fission and fusion was lost, which could explain the mitochondrial accumulation we found. In cases where their fusion is blocked, the mitochondria become dysfunctional and consume cytoplasmic ATP to maintain their membrane potential (Knott and Bossy-Wetzel, 2008; Gomes et al., 2011). To test whether the fission of mitochondria resulted in more mitochondria, we determined the copy number of a cox 2 gene in mtDNA using qPCR, normalized to the $N d u f s 7$ gene in BPH's genomic DNA. However, the relative copy number of the cox2 gene failed to change significantly after the RNAi of NlATG1 (Supplementary Figure S2). Just over 10 years ago, it was suggested that fission events may be indispensable for autophagy (Twig et al., 2008a). Fission is more likely to separate dysfunctional parts, including the mtDNA, from the mitochondrial network (Twig et al., 2008a). As is known, one or more mtDNAs usually exist in a mitochondrion (Rand, 2001), so during the fission process, random separation of mtDNA leads to the separation of defective mtDNAs (Twig et al., 2008b). We presumed that during the inhibition of autophagy in $\mathrm{BPH}$ through the RNAi of NIATG1, the fission of its mitochondria was upregulated, yet their fusion went unaffected, so more damaged mitochondria accumulated and thereby affected the functioning of this insect's mitochondria. However, the mechanism of how RNAi of NlATG1 affected the fission of mitochondria needs to be further explored.

\section{CONCLUSION}

In conclusion, our work here identified an autophagy-related gene of BPH, NlATG1, and revealed both its developmental and spatiotemporal expression profiles. RNA interference and TEM results confirmed that the NIATG1 gene plays a key role in the

\section{REFERENCES}

Araujo, R. N., Santos, A., Pinto, F. S., Gontijo, N. F., Lehane, M. J., and Pereira, M. H. (2006). RNA interference of the salivary gland nitrophorin 2 in the triatomine bug Rhodnius prolixus (Hemiptera: Reduviidae) by dsRNA ingestion or injection. Insect Biochem. Mol. 36, 683-693. doi: 10.1016/j.ibmb.2006.05.012

Arun, S., Liu, L., and Donmez, G. (2016). Mitochondrial biology and neurological biseases. Curr. Neuropharmacol. 14, 143-154. doi: 10.2174/ 1570159x13666150703154541

Bachman, P. M., Bolognesi, R., Moar, W. J., Mueller, G. M., Paradise, M. S., Ramaseshadri, P., et al. (2013). Characterization of the spectrum of insecticidal activity of a double-stranded RNA with targeted activity against Western Corn Rootworm (Diabrotica virgifera virgifera LeConte). Transgenic Res. 22, 1207-1222. doi: 10.1007/s11248-013-9716-5

Baum, J. A., Bogaert, T., Clinton, W., Heck, G. R., Feldmann, P., Ilagan, O., et al. (2007). Control ofcoleopteran insect pests through RNA interference. Nat. Biotechnol. 25, 1322-1326. doi: 10.1038/nbt1359 autophagy of this insect. RNA interference of the NIATG1 gene caused elevated mortality, suggesting that it is a promising target for management of this pest.

\section{DATA AVAILABILITY STATEMENT}

The datasets generated in this study have been deposited in the GenBank database (accession numbers MF169984.1 and MF062504).

\section{AUTHOR CONTRIBUTIONS}

$\mathrm{PH}$ and $\mathrm{XY}$ designed the experiments. FY, CY, YF, and $\mathrm{KP}$ conducted the experiments. $\mathrm{PH}, \mathrm{XY}$, and FY wrote and edited the manuscript.

\section{FUNDING}

This work was supported by the National Natural Science Foundation of China (grant numbers 31672026, 31171860, and 30900944), the Key Research and Development Program of Zhejiang Province, China (grant number 2019C02015), and the Basic Applied Research Project of Zhejiang Province, China (grant numbers LY20C140005, 2012C22041, and LQ18C140002).

\section{ACKNOWLEDGMENTS}

The authors thank the Bio-Ultrastructure Analysis Laboratory of the Analysis Center of Agrobiology and Environmental Sciences, Zhejiang University, for help in the TEM experimental work.

\section{SUPPLEMENTARY MATERIAL}

The Supplementary Material for this article can be found online at: https://www.frontiersin.org/articles/10.3389/fphys. 2019.01622/full\#supplementary-material

Casati, B., Terova, G., Cattaneo, A. G., Rimoldi, S., Franzetti, E., Eguileor, M. D., et al. (2012). Molecular cloning, characterization and expression analysis of ATG1 in the silkworm, Bombyx mori. Gene 511, 326-337. doi: 10.1016/j.gene. 2012.09.086

Cherry, S. (2009). VSV infection is sensed by Drosophila, attenuates nutrient signaling, and thereby activates antiviral autophagy. Autophagy 5, 1062-1063. doi: 10.4161/auto.5.7.9730

Chew, L. H., Lu, S., Liu, X., Li, F. K., Yu, A. Y., Klionsky, D. J., et al. (2015). Molecular interactions of the Saccharomyces cerevisiae Atg1 complex provide insights into assembly and regulatory mechanisms. Autophagy 11, 891-905. doi: 10.1080/15548627.2015.1040972

Chun, Y., and Kim, J. (2018). Autophagy: an essential degradation program for cellular homeostasis and life. Cells 7:278. doi: 10.3390/cells71 20278

Cooper, A. M., Silver, K., Zhang, J., Park, Y., and Zhu, K. Y. (2019). Molecular mechanismsinfluencing efficiency of RNA interference in insects. Pest Manag. Sci. 75, 18-28. doi: 10.1002/ps.5126 
Földvári-Nagy, L., Ari, E., Csermely, P., Korcsmáros, T., and Vellai, T. (2014). Starvation-response may not involve Atg1-dependent autophagy induction in non-unikont parasites. Sci. Rep. 4:5829. doi: 10.1038/srep05829

Franzetti, E., Casartelli, M., D’Antona, P., Montali, A., Romanelli, D., Cappellozza, S., et al. (2016). Midgut epithelium in molting silkworm: a fine balance among cell growth, differentiation, and survival. Arthropod. Struct. Dev. 45, 368-379. doi: 10.1016/j.asd.2016.06.002

Franzetti, E., Huang, Z. J., Shi, Y. X., Xie, K., Deng, X. J., Li, J. P., et al. (2012). Autophagy precedes apoptosis during the remodeling of silkworm larval midgut. Apoptosis 17, 305-324. doi: 10.1016/j.asd.2016.06.002

Ge, L. Q., Zhou, Y. K., Gu, H. T., Wu, Q., Zhou, Z., Zheng, S., et al. (2019). Male selenoprotein F-like (SPF-L) influences female reproduction and population growth in Nilaparvata lugens (Hemiptera: Delphacidae). Front. Physiol. 10:1196. doi: 10.3389/fphys.2019.01196

Gomes, L. C., Benedetto, G. D., and Scorrano, L. (2011). During autophagy mitochondria elongate, are spared from degradation and sustain cell viability. Nat. Cell Biol. 13, 589-598. doi: 10.1038/ncb2220

Hou, Y. C. C., Chittaranjan, S., Barbosa, S. G., McCall, K., and Gorski, S. M. (2008). Effector caspase Dcp-1 and IAP protein Bruce regulate starvationinduced autophagy during Drosophila melanogaster oogenesis. J. Cell Biol. 182, 1127-1139. doi: 10.1083/jcb.200712091

Huang, X., Zheng, C., Liu, F., Yang, C., Zheng, P., Lu, X., et al. (2019). Genetic analyses of the arabidopsis ATG1 kinase complex reveal both kinase-dependent and independent autophagic routes during fixed-carbon starvation. Plant Cell 31, 2973-2995. doi: 10.1105/tpc.19.00066

Huvenne, H., and Smagghe, G. (2010). Mechanisms of dsRNA uptake in insects and potential of RNAi for pest control: a review. J. Insect Physiol. 56, 227-235. doi: 10.1016/j.jinsphys.2009.10.004

Jing, K., and Lim, K. (2012). Why is autophagy important in human diseases? Exp. Mol. Med. 44, 69-72. doi: 10.3858/emm.2012.44.2.028

Khoa, D. B., and Takeda, M. (2012). Expression of autophagy 8 (Atg8) and its role in the midgut and other organs of the greater wax moth, Galleria mellonella, during metamorphic remodelling and under starvation. Insect Mol. Biol. 21, 473-487. doi: 10.1111/j.1365-2583.2012.01152.x

Knott, A. B., and Bossy-Wetzel, E. (2008). Impairing the mitochondrial fission and fusion balance: a new mechanism of neurodegeneration. Ann. N. Y. Acad. Sci. 1147, 283-292. doi: 10.1196/annals.1427.030

Lee, C. Y., Cooksey, B. A. K., and Baehrecke, E. H. (2002). Steroid regulation of midgut cell death during Drosophila development. Dev. Biol. 250, 101-111. doi: 10.1006/dbio.2002.0784

Li, F., Chung, T., and Vierstra, R. D. (2014). AUTOPHAGY-RELATED11 plays a critical role in general autophagy-and senescence-induced mitophagy in arabidopsis. Plant Cell 26, 788-807. doi: 10.1105/tpc.113.120014

Li, H., Khajuria, C., Rangasamy, M., Gandra, P., Fitter, M., Geng, C., et al. (2015). Long dsRNA but not siRNA initiates RNAi in western corn rootworm larvaeand adults. J. Appl. Entomol. 139, 432-445. doi: 10.1111/jen.12224

Liu, H., Jia, Q., Tettamanti, G., and Li, S. (2013). Balancing crosstalk between 20-hydroxyecdysone-induced autophagy and caspase activity in the fat body during Drosophila larval-prepupal transition. Insect Biochem. Mol. 43, 10681078. doi: 10.1016/j.ibmb.2013.09.001

Liu, K., Tang, Q., Fu, C., Peng, J., Yang, H., Li, Y., et al. (2007). Influence of glucose starvation on the pathway of death in insect cell line Sl: apoptosis follows autophagy. Cytotechnology 54, 97-105. doi: 10.1007/s10616-007-9080-5

Liu, S., Ding, Z., Zhang, C., Yang, B., and Liu, Z. (2010). Gene knockdown by intro-thoracic injection of double-stranded RNA in the brown planthopper, Nilaparvata lugens. Insect Biochem. Mol. 40, 666-671. doi: 10.1016/j.ibmb.2010. 06.007

Liu, S., Jaouannet, M., Dempsey, D. M. A., Imani, J., Coustau, C., and Kogel, K. H. (2019). RNA-based technologies for insect control in plant production. Biotechnol. Adv. doi: 10.1016/j.biotechadv.2019.107463 [Epub ahead of print].

Liu, S., Liang, Q. M., Zhou, W. W., Jiang, Y. D., Zhu, Q. Z., Yu, H., et al. (2015). RNA interference of NADPH-cytochrome P450 reductase of the rice brown planthopper, Nilaparvata lugens, increases susceptibility to insecticides. Pest Manag. Sci. 71, 32-39. doi: 10.1002/ps.3760

Liu, Y., Schiff, M., Czymmek, K., Tallóczy, Z., Levine, B., and Dineshkumar, S. P. (2005). Autophagy regulates programmed cell death during the plant innate immune response. Cell 121, 567-577. doi:10.1016/j.cell.2005. 03.007
Livak, K. J., and Schmittgen, T. D. (2001). Analysis of relative gene expression data using real-time quantitative PCR and the 2- $\triangle \triangle$ Ct Method. Methods 25, 402-408. doi: 10.1006/meth.2001.1262

Löffler, A. S., Alers, S., Dieterle, A. M., Keppeler, H., Franz-Wachtel, M., Kundu, M., et al. (2011). Ulk1-mediated phosphorylation of AMPK constitutes a negative regulatory feedback loop. Autophagy 7, 696-706. doi: 10.4161/auto.7.7. 15451

Lőrincz, P., Mauvezin, C., and Juhász, G. (2017). Exploring autophagy in Drosophila. Cells 6:E22. doi: 10.3390/cells6030022

Ma, P., Yun, J., Deng, H., and Guo, M. (2018). Atg1 mediated autophagy suppresses tissue degeneration in pink1/parkin mutants by promoting mitochondrial fission in Drosophila. Mol. Biol. Cell 29:mbcE18040243. doi: 10.1091/mbc.E1804-0243

Manjunatha, G. K. S., Peter, A., Naika, M. B. N., Niranjana, P., and Shamprasad, P. (2018). Identification of in-vitro red fluorescent protein with antipathogenic activity from the midgut of the silkworm (Bombyx mori L.). Protein Peptide Lett. 25, 302-313. doi: 10.2174/0929866525666180115121853

Mao, Y. B., Cai, W. J., Wang, J. W., Hong, G. J., Tao, X. Y., Wang, L. J., et al. (2007). Silencing a cotton bollworm P450 monooxygenase gene by plant-mediated RNAi impairs larval tolerance of gossypol. Nat. Biotechnol. 25, 1307-1313. doi: $10.1038 /$ nbt1352

Mari, M., and Reggiori, F. (2016). Atg9 trafficking in the yeast Saccharomyces cerevisiae. Autophagy 3, 145-148. doi: 10.4161/auto.3608

Mcphee, C. K., and Baehrecke, E. H. (2009). Autophagy in Drosophila melanogaster. BBA Mol. Cell. Res. 1793, 1452-1460. doi: 10.1016/j.bbamcr.2009.02.009

Papinski, D., and Kraft, C. (2016). Regulation of autophagy by signaling through the Atg1/ULK1 complex. J. Mol. Biol. 428, 1725-1741. doi: 10.1016/j.jmb.2016. 03.030

Park, D. S., Lee, S. K., Lee, J. H., Song, M. Y., Song, S. Y., Kwak, D. Y., et al. (2007). The identification of candidate rice genes that confer resistance to the brown planthopper (Nilaparvata lugens) through representational difference analysis. Theor. Appl. Genet. 115, 537-547. doi: 10.1007/s00122-007-0587-0

Rand, D. M. (2001). The units of selection on mitochondrial DNA. Annu. Rev. Ecol. Syst. 32, 415-448. doi: 10.1146/annurev.ecolsys.32.081501.114109

Santana, R. A. G., Oliveira, M. C., Cabral, I., Junior, R. C. A. S., de Sousa, D. R. T., Ferreira, L., et al. (2019). Anopheles aquasalis transcriptome reveals autophagic responses to Plasmodium vivax midgut invasion. Parasit. Vector 12:261. doi: 10.1186/s13071-019-3506-8

Scott, J. G., Michel, K., Bartholomay, L. C., Siegfried, B. D., Hunter, W. B., Smagghe, G., et al. (2013). Towards the elements of successful insect RNAi. J. Insect Physiol. 59:1212. doi: 10.1016/j.jinsphys.2013.08.014

Scott, R. C., Gábor, J., and Neufeld, T. P. (2007). Direct induction of autophagy by Atg1 inhibits cell growth and induces apoptotic cell death. Curr. Biol. 17, 1-11. doi: $10.1016 /$ j.cub.2006.10.053

Shang, L., and Wang, X. (2011). AMPK and mTOR coordinate the regulation of Ulk1 and mammalian autophagy initiation. Autophagy 7, 924-926. doi: 10. 4161/auto.7.8.15860

Suttangkakul, A., Li, F., Chung, T., and Vierstra, R. D. (2011). The ATG1/ATG13 protein kinase complex is both a regulator and a target of autophagic recycling in arabidopsis. Plant Cell 23, 3761-3779. doi: 10.1105/tpc.111.090993

Turner, C. T., Davy, M. W., MacDiarmid, R. M., Plummer, K. M., Birch, N. P., and Newcomb, R. D. (2006). RNA interference in the light brown apple moth, Epiphyas postvittana (Walker) induced by double-stranded RNA feeding. Insect Mol. Biol. 15, 383-391. doi: 10.1111/j.1365-2583.2006.00656.x

Twig, G., Elorza, A., Molina, A. J. A., Mohamed, H., Wikstrom, J. D., Walzer, G., et al. (2008a). Fission and selective fusion govern mitochondrial segregation and elimination by autophagy. EMBO J. 27, 433-446. doi: 10.1038/sj.emboj. 7601963

Twig, G., Hyde, B., and Shirihai, O. S. (2008b). Mitochondrial fusion, fission and autophagy as a quality control axis: the bioenergetic view. BBA Bioenerg. 1777, 1092-1097. doi: 10.1016/j.bbabio.2008.05.001

Van der Vaart, A., Mari, M., and Reggiori, F. (2008). A picky eater: exploring the mechanisms of selective autophagy in human pathologies. Traffic 9, 281-289. doi: 10.1111/j.1600-0854.2007.00674.x

Wang, B., and Kundu, M. (2017). Canonical and noncanonical functions of ULK/Atg1. Curr. Opin. Cell Biol. 45, 47-54. doi: 10.1016/j.ceb.2017.02.011

Wang, L. L., Wang, X. R., Wei, X. M., Huang, H., Wu, J. X., Chen, X. X., et al. (2016). The autophagy pathway participates in resistance to tomato yellow leaf curl 
virus infection in whiteflies. Autophagy 12, 1560-1574. doi: 10.1080/15548627. 2016.1192749

Wang, W., Li, K., Wan, P., Lai, F., Fu, Q., and Zhu, T. (2017). Ras-like family small GTPases genes in Nilaparvata lugens: identification, phylogenetic analysis, gene expression and function in nymphal development. PLoS One 12:e172701. doi: 10.1371/journal.pone.0172701

Wang, Y., Chen, J., Zhu, Y. C., Ma, C., Huang, Y., and Shen, J. (2008). Susceptibility to neonicotinoids and risk of resistance development in the brown planthopper, Nilaparvata lugens (Stål) (Homoptera: Delphacidae). Pest Manag. Sci. 64, 12781284. doi: $10.1002 /$ ps. 1629

Wang, Y., Zhang, H., Li, H., and Miao, X. (2011). Second-generation sequencing supply an effective way to screen RNAi targets in large scale for potential application in pest insect control. PLoS One 6:e18644. doi: 10.1371/journal. pone.0018644

Waris, M. I, Younas, A., Ul Qamar, M. T., Hao, L., Ameen, A., Ali, S., et al. (2018). Silencing of chemosensory protein gene NlugCSP8 by RNAi induces declining behavioral responses of Nilaparvata lugens. Front. Physiol. 9:379. doi: 10.3389/fphys.2018.00379

Whyard, S., Singh, A. D., and Wong, S. (2009). Ingested double-stranded RNAs can act asspecies-specific insecticides. Insect Biochem. Mol. 39, 824-832. doi: 10.1016/j.ibmb.2009.09.007

Wong, P. M., Puente, C., Ganley, I. G., and Jiang, X. (2013). The ULK1 complex: sensing nutrient signals for autophagy activation. Autophagy 9, 124-137. doi: 10.4161/auto.23323

Wu, W., Wei, W., Ablimit, M., Ma, Y., Fu, T., Liu, K., et al. (2011). Responses of two insect cell lines to starvation: autophagy prevents them from undergoing apoptosis and necrosis, respectively. J. Insect Physiol. 57, 723-734. doi: 10.1016/ j.jinsphys.2011.02.008

Xue, W. H., Xu, N., Yuan, X. B., Chen, H. H., Zhang, J. L., Fu, S. J., et al. (2018). CRISPR/Cas9-mediated knockout of two eye pigmentation genes in the brown planthopper, Nilaparvata lugens (Hemiptera: Delphacidae). Insect Biochem. Mol. 93, 19-26. doi: 10.1016/j.ibmb.2017.12.003
Yu, X. D., Liu, Z. C., Huang, S. L., Chen, Z. Q., Sun, Y. W., Duan, P. F., et al. (2016). RNAi-mediated plant protection against aphids. Pest Manag. Sci. 72, 1090-1098. doi: 10.1002/ps.4258

Yuan, M., Lu, Y., Zhu, X., Wan, H., Shakeel, M., Zhan, S., et al. (2014). Selection and evaluation of potential reference genes for gene expression analysis in the brown planthopper, Nilaparvata lugens (Hemiptera: Delphacidae) using reverse-transcription quantitative PCR. PLoS One 9:e86503. doi: 10.1371/ journal.pone.0086503

Yue, Y. J., Tang, X. D., Xu, L., Yan, W., Li, Q. L., Xiao, S. Y., et al. (2015). Early responses of silkworm midgut to microsporidium infection - A digital gene expression analysis. J. Invertebr. Pathol. 124, 6-14. doi: 10.1016/j.jip.2014.10. 003

Zhan, L., Li, J., and Wei, B. (2018). Autophagy in endometriosis: friend or foe? Biochem. Biophys. Res. 495, 60-63. doi: 10.1016/j.bbrc.2017.10.145

Zhang, N., Yang, Y., Lu, H., Xiang, Y., Huang, X., Hu, R., et al. (2016). Spodoptera litura autophagy-related protein 1 interacts with autophagy-related protein 5 and enhances its degradation. Insect Mol. Biol. 26, 190-203. doi: 10.1111/imb. 12284

Zhu, J., Hao, P., Lu, C., Ma, Y., Feng, Y., and Yu, X. (2017). Expression and RNA interference of ribosomal protein L5 gene in Nilaparvata lugens (Hemiptera: Delphacidae). J. Insect Sci. 17:73. doi: 10.1093/jisesa/iex047

Conflict of Interest: The authors declare that the research was conducted in the absence of any commercial or financial relationships that could be construed as a potential conflict of interest.

Copyright (c) $2020 \mathrm{Yu}, \mathrm{Hao}, \mathrm{Ye}$, Feng, Pang and Yu. This is an open-access article distributed under the terms of the Creative Commons Attribution License (CC BY). The use, distribution or reproduction in other forums is permitted, provided the original author(s) and the copyright owner(s) are credited and that the original publication in this journal is cited, in accordance with accepted academic practice. No use, distribution or reproduction is permitted which does not comply with these terms. 